\title{
On Reactive Power Flow and Voltage Stability in Microgrids
}

\author{
Basilio Gentile, John W. Simpson-Porco, Florian Dörfler, Sandro Zampieri, and Francesco Bullo
}

\begin{abstract}
This paper focuses on reactive power flow and voltage stability in electrical grids. We provide novel analytical understanding of the solutions to the classic nonlinear polynomial equations describing the decoupled reactive power flow. As of today, solutions to these equations can be found only via numerical methods. Yet an analytical understanding would enable rigorous design of future electrical grids. This paper has two main contributions. First, for sufficiently-high reference voltages, we guarantee the existence of a high-voltage solution for the reactive power flow equations and provide its approximate analytical expression. The approximation error is bounded in terms of network topology and parameters. Second, we consider a recently-proposed droop control strategy for voltage stabilization in a microgrid equipped with inverters. For sufficiently-high reference voltages, we prove the existence and the exponential stability of a high-voltage fixed point of the closed-loop dynamics. We provide an approximate expression for this fixed point and find the limiting value of the approximation error for high reference voltages. Finally, we validate the accuracy of our approximations through numerical simulation of the IEEE 37 standard test case.
\end{abstract}

\section{INTRODUCTION}

The power flow equations model the relationships among bus power injections, power demands, and bus voltages and angles in a power network. They are the heart of most system-planning and operational studies and also the starting point for transient and dynamic stability studies. They constitute a set of coupled equations with trigonometric and polynomial non-linearities, and the solution space admits a rich and complex phenomenology [1], [2]. Conditions for the existence and exact expression of the solutions have been derived for the case of a radial grid [3], while for a general network only conservative conditions have been proposed [4]-[6]. This lack of sharp results has motivated approximate solutions. Of particular interest is [7], where an approximate solution to the reactive power flow equations was developed for electrical networks connected to a larger parent grid at a single Point of Common Coupling, such as typical distribution networks. While this analytic approximation is potentially powerful, its use is limited in more general electrical networks due to the presence of multiple fixedvoltage buses. Analytic results aside, the current standard

This work was supported in part by NSF Grant 1135819 .

B. Gentile and S. Zampieri are with the Department of Information Engineering, University of Padova, Italy \{gentileb, zampi\}@dei.unipd.it. B. Gentile is also with the Technical University of Denmark, Lyngby, Denmark. J. W. Simpson-Porco and F. Bullo are with the Department of Mechanical Engineering and the Center for Control, Dynamical Systems and Computation, University of California at Santa Barbara \{johnwsimpsonporco, bullo\}@engineering . ucsb. edu. F. Dörfler is with the Department of Electrical Engineering, University of California at Los Angeles. dorflerdseas.ucla.edu. for power flow solution is numerical simulation [8], and the power industry invests considerable effort in simulating thousands of power flow equations for large grids. This motivates the importance of a deeper analytic insight into the problem.

A classic approach [4], [5] to the analysis of the power flow equations is to study the active and the reactive power equations separately under mild decoupling assumptions which are usually satisfied under regular system operation [9]. After the decoupling, the phase angles become the only variables appearing in the active power equations, while the voltage magnitudes become the only variables in the reactive ones. We focus our attention on the resulting reactive power flow equations: these are a system of quadratic equations in the voltage magnitudes at the buses. Despite the simpler problem formulation, no sharp analytic answers pertaining to the existence of solutions are known to date [4]-[6].

The first contribution of this paper is the extension of the approximate load flow solution proposed in [7] to networks with multiple fixed-voltage buses. In particular, we present the result as an approximate solution to the decoupled reactive power flow equations. The resulting solution can be viewed as the reactive power counterpart of the DC load flow approximation for the active power flow [10]. The classic DC load flow approximation expresses the solution to the non-linear active flow equations as a linear combination of the active powers at the buses. The linear coefficients only depends on the network parameters. The approximate solution that we propose for the reactive power flow is the sum of two main terms: the first one is similar to the DC approximation, as it is a linear combination of the reactive powers at the buses; the linear coefficients only depend on the network parameters. The second term consists of a constant high-voltage value for each bus, and it is related to the general and well accepted idea that strongly-clustered high-voltage solutions of the reactive flow equations are the desired stable solutions [11].

In the second part of the paper we focus on the stability of a droop control strategy in an islanded microgrid. Microgrids are low-voltage electrical distribution networks, heterogeneously composed of distributed generation, load, and managed autonomously from the larger primary network. Power sources in microgrids generate either variable frequency $\mathrm{AC}$ power or DC power, and are interfaced with a synchronous $\mathrm{AC}$ microgrid via power electronic DC/AC inverters. In islanded operation, it is through these inverters that actions must be taken to ensure synchronization, voltage stability, power balance and load sharing in the network [12]. We consider the problem of voltage stabilization; that is, 
keeping the average voltage level in the network high, and keeping the total voltage profile roughly uniform. This is a crucial aspect of microgrid control, as the relatively low voltage levels and uncompensated loads in microgrids put the network at risk for voltage instability and collapse [2]. In the last two decades the $E-Q$ voltage-droop controller has become the tool commonly used for these tasks [13]. Despite the wide-spread adoption of the $E-Q$ voltagedroop controller, few analytic results are available about its closed-loop performance. Specifically, to the best of our knowledge, no results are available on the existence and locations of the equilibria of the closed-loop network. This paper considers the quadratic droop controller proposed by [14]. This modified version of the standard $E-Q$ droop controller reproduces the inherently quadratic and asymmetric nature of the reactive power flow equations and facilitates an analytic treatment. Our previous work [14] characterizes the existence, stability and location of the equilibrium point for a purely-inductive (lossless) network with parallel topology. In this work, we consider networks with arbitrary topology and with arbitrary heterogeneous (resistive and inductive) impedences; by applying the approximation method proposed for the reactive power flow equations, this paper establishes the existence and the stability of a high-voltage fixed point and provides an approximate expression for its location.

This paper is organized as follows. In what follows we introduce the reactive power flow equations. Section II defines the approximate solution to the reactive power flow equations. Section III applies the approximation method to droop-controlled microgrid. Section IV reports some numerical studies. Section V contains our concluding remarks.

\section{Notation and network model}

Given a finite set $\mathcal{V}$, let $|\mathcal{V}|$ denote its cardinality. Let $\mathbb{1}$ denote the vector of all ones, $\mathbf{0}$ a matrix of all zeros; their dimension is not specified as it is understandable from the context. Let $\left[x_{i}\right]_{i \in \mathcal{V}}$ be an alternative notation for the vector $x$, with indices in the set $\mathcal{V}$. Let $\operatorname{diag}(x)$ denote the diagonal matrix whose main diagonal is the vector $x$ and $\operatorname{diag}^{-1}(x)$ its inverse, when defined. Given the vectors $x$ and $y$, we write $x>y$ (resp. $x \geq y$ ) if $x_{i}>y_{i}$ (resp. $x_{i} \geq y_{i}$ ), for all $i \in I$. For $a \in \mathbb{C}, a^{*}$ denotes the complex-conjugate of $a$.

A power network in synchronous steady-state is modeled as a connected, undirected and complex-weighted graph $G(\mathcal{V}, \mathcal{E}, Y)$, where $\mathcal{V}=\{1, \ldots, n\}$ is the set of nodes (or buses) and $\mathcal{E} \subset \mathcal{V} \times \mathcal{V}$ is the set of edges (or branches). Since the graph is undirected, if $(i, j) \in \mathcal{E}$, then it is also $(j, i) \in \mathcal{E}$. To each edge $(i, j)$ we assign an admittance $W_{i j}=W_{j i} \in \mathbb{C}$; we define the off-diagonal elements of the complex-valued Laplacian matrix $Y$ by $Y_{i j}=-W_{i j}, i \neq j$, while the diagonal elements are defined by $Y_{i i}=-\sum_{i \neq j} Y_{i j}$. To each node $i \in \mathcal{V}$ we assign a phasor voltage $U_{i}=E_{i} e^{j \theta_{i}} \in \mathbb{C}$, a phasor current $I_{i} \in \mathbb{C}$, and a power injection $S_{i}=$ $P_{i}+j Q_{i} \in \mathbb{C}$, whose real part $P_{i} \in \mathbb{R}$ is the active power and imaginary part $Q_{i} \in \mathbb{R}$ is the reactive power. In vector notation, Kirchoff's current law and Ohm's law give the current-balance relation $I=Y U$. Moreover, power, voltage and current at each node are related through: $S_{i}=U_{i} I_{i}^{*}$. Combining the last two equations in vector notation,

$$
P+j Q=\operatorname{diag}(U)(Y U)^{*},
$$

which in components reduces to the active and reactive power equations, that is,

$$
\begin{aligned}
P_{i}= & \sum_{j=1}^{n} \mathfrak{I m}\left(Y_{i j}\right) E_{i} E_{j} \sin \left(\theta_{i}-\theta_{j}\right) \\
& +\sum_{j=1}^{n} \mathfrak{R e}\left(Y_{i j}\right) E_{i} E_{j} \cos \left(\theta_{i}-\theta_{j}\right), \quad i \in \mathcal{V},
\end{aligned}
$$

and

$$
\begin{aligned}
Q_{i}= & -\sum_{j=1}^{n} \mathfrak{I m}\left(Y_{i j}\right) E_{i} E_{j} \cos \left(\theta_{i}-\theta_{j}\right) \\
& +\sum_{j=1}^{n} \mathfrak{R e}\left(Y_{i j}\right) E_{i} E_{j} \sin \left(\theta_{i}-\theta_{j}\right), \quad i \in \mathcal{V} .
\end{aligned}
$$

During regular power system operation the solutions to (1) usually [9], [10] satisfy $\left|\theta_{i}-\theta_{j}\right| \ll 1$ for each $(i, j) \in \mathcal{E}$. We assume from now on that $\theta_{i}=\theta_{j}$ for each $(i, j) \in \mathcal{E}$. Under this condition, the reactive power flow equations (RPFE) (3) can be written in compact vector notation as

$$
Q=\operatorname{diag}(E) L E,
$$

where $L=-\mathfrak{I m}(Y)$ is a Laplacian matrix (with non-positive off-diagonal terms and zero row sums) since the susceptance of each resistive and inductive line is negative. As standard in load flow studies and power system stability analysis, we model loads as stiff constant-power demands [9].

\section{ApProximate SOlution to the ReACtive POWER FLOW EQUATIONS}

In this section we partition the network nodes as $\mathcal{V}=$ $\left\{\mathcal{V}_{L}, \mathcal{V}_{S}\right\}$ corresponding to loads and sources (or generators). We require that the voltage magnitudes at these sources are regulated to constant and predetermined values. The typical example of such a network is a transmission-level grid consisting of loads and $P E$-generation sources such as synchronous generators. The voltage magnitude vector and the Laplacian $L$ inherit the partitioning as

$$
E=\left[\begin{array}{l}
E_{L} \\
E_{S}
\end{array}\right], \quad L=\left[\begin{array}{ll}
L_{L L} & L_{L S} \\
L_{S L} & L_{S S}
\end{array}\right] .
$$

With this in mind, equation (4) becomes

$$
\left[\begin{array}{l}
Q_{L} \\
Q_{S}
\end{array}\right]=\operatorname{diag}\left(E_{L}, E_{S}\right)\left[\begin{array}{ll}
L_{L L} & L_{L S} \\
L_{S L} & L_{S S}
\end{array}\right]\left[\begin{array}{c}
E_{L} \\
E_{S}
\end{array}\right] .
$$

We assume that the source voltages $E_{S}$ are fixed and no constraints are imposed on the sources power injections $Q_{S}$, that is, the sources are $P E$-buses [9]. Hence, the second block of equations in (5) can be thought of as determining $Q_{S}$ as a function of the load voltages $E_{L}$. Thus, the equations (5) reduce to their first block:

$$
Q_{L}=\operatorname{diag}\left(E_{L}\right)\left[\begin{array}{ll}
L_{L L} & L_{L S}
\end{array}\right]\left[\begin{array}{c}
E_{L} \\
E_{S}
\end{array}\right] .
$$

The variables in these $\left|\mathcal{V}_{L}\right|$ equations (6) are the $\left|\mathcal{V}_{L}\right|$ load voltages $E_{L}$. In other words, these equations, if solvable, 
determine $E_{L}$ as a function of the remaining constant source voltages and network parameters.

In general the system of quadratic equations (6) is not solvable analytically. The classic example of a two node network nicely illuminates some of the general features of these equations and motivates our subsequent approximation.

Example II.1 (Two node network). Consider a network with two nodes connected through an inductive line with susceptance $-\ell$. One node is a load with reactive power demand $q$, while the other node is a source with fixed voltage magnitude $E_{N}>0$. Denoting by $e$ the voltage magnitude at the load, (6) reduces to

$$
q=\ell e\left(e-E_{N}\right) .
$$

If

$$
q \geq-q_{\mathrm{crit}}:=-\frac{1}{4} \ell E_{N}^{2},
$$

then equation (7) admits two real-valued solutions, given by

$$
e_{1,2}=E_{N}\left(\frac{1}{2} \pm \frac{1}{2} \sqrt{1+\frac{q}{q_{\text {crit }}}}\right) .
$$

If $\left|q / q_{\text {crit }}\right| \ll 1$, the Taylor expansion $\left(\sqrt{1+x} \simeq 1+\frac{1}{2} x\right)$ leads to the approximate expressions:

$$
e_{1} \simeq E_{N}+\frac{q}{\ell E_{N}}, \quad e_{2} \simeq-\frac{q}{\ell E_{N}} .
$$

The solution $e_{1}$ is the desired one in practice, as it corresponds to a high-voltage low-current configuration for the network, resulting in low power losses. In particular, the solution can be interpreted as being roughly $E_{N}$, with a correction term linear in the power demand, scaled inversely by both $E_{N}$ and the line susceptance.

We now build further on the motivation of Example II.1 and offer some intuitive derivations on how to generalize the example. We set $E_{N}:=\min _{i \in \mathcal{V}_{S}} E_{i}$ and define the vector $\eta$ so that the voltages can be decomposed into $E=E_{N}(\mathbb{1}+\eta)$. As in the example, we are interested in the high-voltage solution to the power flow equations and, moreover, we are interested in solutions with uniform voltages. High and uniform voltages correspond to the regime where $E_{N} \gg 1$ and $\eta \ll 1$. In this regime, equation (6) becomes

$$
\begin{aligned}
Q_{L} & =E_{N} \operatorname{diag}\left(\mathbb{1}+\eta_{L}\right)\left[\begin{array}{ll}
L_{L L} & L_{L S}
\end{array}\right] E_{N}(\mathbb{1}+\eta) \\
& =E_{N}^{2}\left(\left[\begin{array}{ll}
L_{L L} & L_{L S}
\end{array}\right] \eta+\operatorname{diag}\left(\eta_{L}\right)\left[\begin{array}{ll}
L_{L L} & L_{L S}
\end{array}\right] \eta\right) \\
& \simeq E_{N}^{2}\left(L_{L L} \eta_{L}+L_{L S} \eta_{S}\right),
\end{aligned}
$$

where the second equality holds because $\mathbb{1}$ is in the kernel of $L$, and the last approximation neglects the quadratic term in $\eta$. Solving (10) for $\eta_{L}$, we obtain the following approximate solution

$$
\begin{aligned}
E_{L} & =E_{N}\left(\mathbb{1}+\eta_{L}\right) \\
& \simeq E_{N} \mathbb{1}-E_{N} L_{L L}^{-1} L_{L S} \eta_{S}+\frac{1}{E_{N}} L_{L L}^{-1} Q_{L} .
\end{aligned}
$$

Looking back at Example II.1, we see how the first order expansion that led to the solution $e_{1}$ in equation (9) corre- sponds exactly to the approximation (11). Building on this intuitive derivations, we now state our first rigorous result, which extends the work carried out in [7] to transmissionlevel networks with multiple generating sources. The proof of the following theorem extends the proof strategy in [7] and uses arguments of multivariate analysis along with the implicit function theorem. Due to space constraints, the proof is not be reported here.

Theorem II.2 (Approximate solution to the RPFE). Consider the reactive power balance equations (6), define $E_{N}:=$ $\min _{i \in \mathcal{V}_{S}} E_{i}$ as the source baseline voltage, and let $\eta_{S}$ be the source voltage spread $E_{S}=E_{N}\left(\mathbb{1}+\eta_{S}\right)$. Define the approximate load voltage

$$
E_{L, \text { approx }}:=E_{N} \mathbb{1}-E_{N} L_{L L}^{-1} L_{L S} \eta_{S}+\frac{1}{E_{N}} L_{L L}^{-1} Q_{L} .
$$

Then there exists a minimum voltage $E_{N}^{\min }$ such that, for all $E_{N}>E_{N}^{\min }$, a high-voltage solution of equation (6) exists and is given by

$$
E_{L}=E_{L, \text { approx }}+\frac{1}{E_{N}} g_{L} \simeq E_{L, \text { approx }},
$$

where the error term $g_{L}$ satisfies

$$
\left\|g_{L}\right\|_{2} \leq \alpha\left\|\eta_{S}\right\|_{2}\left\|Q_{L}\right\|_{2}+\beta \frac{1}{E_{N}^{2}}\left\|Q_{L}\right\|_{2}^{2},
$$

where $\alpha$ and $\beta$ are functions of the network parameters:

$$
\alpha=4 \sqrt{2}\left\|L_{L L}^{-1} L_{L S}\right\|_{2}\left\|L_{L L}^{-1}\right\|_{2}, \quad \beta=4 \sqrt{2}\left\|L_{L L}^{-1}\right\|_{2}^{2} .
$$

Remark II.3 From equation (13) one sees that asymptotically, the error term $g_{L}$ vanishes as the source voltage baseline $E_{N}$ becomes large and the source voltage spread $\eta_{S}$ diminishes. Indeed, this regime is the practically relevant case occurring in regular power system operation, and this qualitative behavior of the error term agrees with classic power systems intuition [6].

While the bound in (13) is quite conservative for any given network, the numerical simulations reported Section IV indicate that the error term $g_{L}$ is much smaller than the theoretical upper bound (13), and thus the approximation (12) is extremely accurate.

\section{APPliCATION TO THE QUADRATIC DROOP CONTROLLER}

In this section, we consider the problem of voltage stabilization in an inverter-based microgrid. We partition the set of nodes in the microgrid as $\mathcal{V}=\left\{\mathcal{V}_{L}, \mathcal{V}_{I}\right\}$, where $\mathcal{V}_{L}$ are loads and $\mathcal{V}_{I}$ are inverters with power injections governed by the quadratic droop controller [14]. This recently proposed controller adjusts the inverter voltage magnitude according to

$$
\tau_{i} \dot{E}_{i}=-C_{i} E_{i}\left(E_{i}-E_{i}^{R}\right)-Q_{i}, \quad i \in \mathcal{V}_{I},
$$

where $\tau_{i}, C_{i}>0$ are fixed controller parameters, and $E_{i}^{R}$ is a fixed reference voltage. If the inverter $i$ injects no reactive power, the equilibrium voltage of (14) is $E_{i}^{R}$. By combining 
the reactive power flow equations at the load (6) and the controller (14), we obtain the differential-algebraic system

$$
\left[\begin{array}{c}
\mathbf{0} \\
\tau \dot{E}_{I}
\end{array}\right]=\left[\begin{array}{c}
Q_{L} \\
C \operatorname{diag}\left(E_{I}\right)\left(E_{R}-E_{I}\right)
\end{array}\right]-\operatorname{diag}(E) L E,
$$

where $\tau=\operatorname{diag}\left(\left[\tau_{i}\right]_{i \in \mathcal{V}_{I}}\right)$ and $C=\operatorname{diag}\left(\left[C_{i}\right]_{i \in \mathcal{V}_{I}}\right)$ are diagonal matrices, while $E_{R}=\left[E_{i}^{R}\right]_{i \in \mathcal{V}_{R}}$ is the vector of the reference voltages. We point out that while in Section II the voltages $E_{S}$ were considered to be fixed, now due to the introduction of the quadratic droop controller the voltages $E_{I}$ in (15) are variables of the system; hence the variables are now $E_{L}$ and $E_{I}$. The goal of this section is to study whether the differential-algebraic system (15) possesses a fixed point, to determine its stability, and find an approximate expression.

Remark III.1 (Network interpretation of quadratic droop controller). Comparing (14) and the right-hand side of (7), the term $C_{i} E_{i}\left(E_{i}-E_{i}^{R}\right)$ in (14) can be interpreted as the reactive power injected from inverter $i$ to a fictitious node of voltage $E_{i}^{R}$ through a line of susceptance $-C_{i}$. Guided by this intuition, we consider an extended network (Figure 1) where we introduce the set of reference nodes $\mathcal{V}_{R}$ and we connect each node $i \in \mathcal{V}_{I}$ to the corresponding reference node $i \in \mathcal{V}_{R}$ (with voltage $E_{i}^{R}$ ) through a line of susceptance $-C_{i}$. The voltage vector and Laplacian matrix of the extended network are

$$
\tilde{E}=\left[\begin{array}{c}
E_{L} \\
E_{I} \\
E_{R}
\end{array}\right], \quad \tilde{L}=\left[\begin{array}{ccc}
L_{L L} & L_{L I} & \mathbf{0} \\
L_{I L} & L_{I I}+C & -C \\
\mathbf{0} & -C & C
\end{array}\right],
$$

where the diagonal matrix $C$ represents the new connections established between inverters $\mathcal{V}_{I}$ and reference nodes $\mathcal{V}_{R}$.



Fig. 1. The equivalence between the original network (top) which consists of an inverter (blue square) $\square$ feeding a load o, and the extended network (bottom) with an additional fictitious node $\diamond$ held at constant voltage $E_{R}$.

From (16) we can compute the reactive power at the inverters in the extended network, which we denote by $\tilde{Q}_{I}$ :

$$
\begin{aligned}
\tilde{Q}_{I} & =\operatorname{diag}\left(E_{I}\right)\left[\begin{array}{lll}
L_{I L} & L_{I I}+C & -C
\end{array}\right] \tilde{E} \\
& =C \operatorname{diag}\left(E_{I}\right)\left(E_{I}-E_{R}\right)+Q_{I},
\end{aligned}
$$

Comparing (17) and (14), the quadratic droop controller can now be expressed as

$$
\tau \dot{E}_{I}=-\tilde{Q}_{I}
$$

Using (18) we can write the differential-algebraic system (15) on the extended network equivalently as

$$
\begin{aligned}
{\left[\begin{array}{l}
Q_{L} \\
\tilde{Q}_{I}
\end{array}\right] } & =\operatorname{diag}\left(E_{L}, E_{I}\right)\left[\begin{array}{ccc}
L_{L L} & L_{L I} & 0 \\
L_{I L} & L_{I I}+C & -C
\end{array}\right] \tilde{E} \\
\tau \dot{E}_{I} & =-\tilde{Q}_{I} .
\end{aligned}
$$

We emphasize that (19) and (15) are equivalent representations of the microgrid with quadratic droop control at the inverters.

The equations (19a) have the same structure as the original load reactive power flow equation (6), and in Theorem II. 2 we introduced an approximate solution to equation (6). We can now follow a similar path and apply the same approximation to equation (19a) to find an approximate solution for the voltages $E_{L}$ and $E_{I}$, while $E_{R}$ is considered to be fixed. We can then use this solution to study the stability of the unique equilibrium $\tilde{Q}_{I}=\mathbf{0}$ of (19b). More precisely, we perform a change of coordinates in equation (19), using the approximation errors as the new system variables. In doing so, we facilitate the analysis of the fixed points of the system (19).

In order to state our main result, we define the reference baseline voltage $\tilde{E}_{N}$ by

$$
\tilde{E}_{N}:=\min _{i \in \mathcal{V}_{R}} E_{i}^{R},
$$

and the reference voltage spread $\tilde{\eta}$ by

$$
E_{R}=\tilde{E}_{N}(\mathbb{1}+\tilde{\eta})
$$

Note that $\tilde{\eta} \geq \mathbf{0}$. We define the inverse $X$ of the truncated Laplacian matrix by

$$
X=\left[\begin{array}{cc}
X_{L L} & X_{L I} \\
X_{I L} & X_{I I}
\end{array}\right]:=\left[\begin{array}{cc}
L_{L L} & L_{L I} \\
L_{I L} & L_{I I}+C
\end{array}\right]^{-1},
$$

and the hybrid matrix

$$
M=\left[\begin{array}{c}
M_{L} \\
M_{I}
\end{array}\right]:=-\left[\begin{array}{cc}
L_{L L} & L_{L I} \\
L_{I L} & L_{I I}+C
\end{array}\right]^{-1}\left[\begin{array}{c}
\mathbf{0} \\
-C
\end{array}\right]=\left[\begin{array}{c}
X_{L I} \\
X_{I I}
\end{array}\right] C .
$$

We are now ready to state the main result of this section.

Theorem III.2 (Existence and Stability of the Fixed Point). There exists a minimum reference baseline voltage $\tilde{E}_{N}^{\min }$ such that for all $\tilde{E}_{N}>\tilde{E}_{N}^{\min }$ the differential-algebraic system (15) has a locally exponentially stable high-voltage fixed point given by

$$
\left[\begin{array}{c}
E_{L}^{e q} \\
E_{I}^{e q}
\end{array}\right]=E_{N} \mathbb{1}+E_{N} M \tilde{\eta}+\frac{1}{E_{N}}\left[\begin{array}{c}
X_{L L} \\
X_{I L}
\end{array}\right] Q_{L}+\frac{1}{E_{N}} g^{\mathrm{eq}}
$$

where the term $g^{\mathrm{eq}}$ becomes constant as the source baseline voltage $E_{N}$ increases, that is,

$$
\lim _{E_{N} \rightarrow \infty} g^{\mathrm{eq}}=-\left[\begin{array}{c}
X_{L L} \\
X_{I L}
\end{array}\right] \operatorname{diag}^{-1}\left(\mathbb{1}+M_{L} \tilde{\eta}\right) \operatorname{diag}\left(M_{I} \tilde{\eta}\right) Q_{L}
$$

Theorem III.2 takes inspiration from Theorem II.2 but addresses a different problem. While Theorem II.2 gives an 
approximate solution of the algebraic equation (6), Theorem III. 2 provides the approximate expression of a fixed point of a differential-algebraic system, and studies its stability. The proof of Theorem III.2 is considerably more complicated than that of Theorem II.2, and we will present it shortly. Note that the two theorems characterize differently the error terms $g$ : Theorem II.2 proposes a bound on the error term $g$ in terms of the network parameters, while Theorem III.2 determines the asymptotic behavior of the error term $g^{\mathrm{eq}}$. However, setting $g^{\text {eq }}=\mathbf{0}$ in equation (21) gives an approximate expression for the solution of (19a). The approximate expression is the same we introduced in Theorem II.2, except that here it is applied to an extended network with $\tilde{Q}_{I}=\mathbf{0}$. The close relationship between the two theorems allows us to verify the accuracy of the approximation (12) of Theorem II.2 by only performing a numerical analysis on the system (19) (see Section IV).

Proof To streamline the presentation, we break the proof into the following three parts:

1) change of variables and simplifications;

2) implicit function theorem for large $\tilde{E}_{N}$;

3) local stability analysis of fixed point.

Part 1 (Change of variables and simplifications): Consider the linear change of variables from $E_{L}$ and $E_{I}$ to $g_{L}$ and $g_{I}$ defined by

$$
\left[\begin{array}{c}
E_{L} \\
E_{I}
\end{array}\right]=E_{N} \mathbb{1}+E_{N}\left[\begin{array}{c}
M_{L} \\
M_{I}
\end{array}\right] \tilde{\eta}+\frac{1}{E_{N}} X\left[\begin{array}{l}
Q_{L} \\
\tilde{Q}_{I}
\end{array}\right]+\frac{1}{E_{N}}\left[\begin{array}{c}
g_{L} \\
g_{I}
\end{array}\right] .
$$

Let us define the following quantities and shorthands:

$\varepsilon:=\frac{1}{\tilde{E_{N}}} g:=\left[\begin{array}{c}g_{L} \\ g_{I}\end{array}\right] \quad Q:=\left[\begin{array}{c}Q_{L} \\ \tilde{Q}_{I}\end{array}\right] \quad \mathcal{L}:=\left[\begin{array}{cc}L_{L L} & L_{L I} \\ L_{I L} & L_{I I}+C\end{array}\right]$.

The matrix $\mathcal{L}$ is a strictly diagonally dominant $M$-matrix, and hence is positive definite (see [15, Corollary 6.2.27]). As a consequence, $\mathcal{X}=\mathcal{L}^{-1}$ as defined by (20) — along with its principal sub-block $X_{I I}$ - are also positive definite. Since $\mathcal{L}$ is a non-singular and irreducible M-matrix, its inverse $X$ is positive [16, Theorem A]. Since $\tilde{\eta}_{i}>0$ and $C_{i}>0$, these properties of $X$ imply that

$$
\mathbb{1}+\left[\begin{array}{c}
M_{L} \\
M_{I}
\end{array}\right] \tilde{\eta}=\mathbb{1}+\left[\begin{array}{c}
X_{L I} \\
X_{I I}
\end{array}\right] C \tilde{\eta}>\mathbf{0}
$$

Applying the change of variables in (19) and performing some simple calculations, we compute that

$$
\left[\begin{array}{ccc}
L_{L L} & L_{L I} & \mathbf{0} \\
L_{I L} & L_{I I}+C & -C
\end{array}\right] \tilde{E}=\varepsilon Q+\varepsilon \mathcal{L} g,
$$

Combining the change of variables (which are now $g_{L}, g_{I}, \tilde{Q}_{I}$ ) and the equality (24), we can reformulate the differential-algebraic system (19) as

$$
\begin{aligned}
& Q=\left(\frac{1}{\varepsilon} \operatorname{diag}(\mathbb{1}+M \tilde{\eta})+\varepsilon \operatorname{diag}(\mathcal{X} Q+g)\right)(\varepsilon Q+\varepsilon \mathcal{L} g) \\
& -\tilde{Q}_{I}=\frac{d}{d t}\left(\frac{1}{\varepsilon}\left(\mathbb{1}+M_{I} \tilde{\eta}\right)+\varepsilon\left(X_{I L} Q_{L}+X_{I I} \tilde{Q}_{I}+g_{I}\right)\right),
\end{aligned}
$$

where without loss of generality we assumed $\tau=I$. Expanding the above equations and performing some simple computations, we obtain the simplified system

$$
\begin{aligned}
\mathbf{0} & =\operatorname{diag}(\mathbb{1}+M \tilde{\eta}) \mathcal{L} g+\operatorname{diag}(M \tilde{\eta}) Q+\varepsilon^{2} h \\
-\tilde{Q}_{I} & =\varepsilon X_{I I} \dot{\tilde{Q}}_{I}+\varepsilon \dot{g}_{I},
\end{aligned}
$$

where we have used $\mathcal{L} \mathcal{X}=I$, the fact that $\tilde{\eta}$ and $Q_{L}$ are constant in time, and we have defined

$h\left(g_{L}, g_{I}, \tilde{Q}_{I}\right):=\operatorname{diag}(\mathcal{X} Q)(Q+\mathcal{L} g)+\operatorname{diag}(g)(Q+\mathcal{L} g)$.

Part 2 (Implicit function theorem for large $\tilde{\boldsymbol{E}}_{N}$ ): The algebraic equation (25a) has the form

$$
\mathbf{0}=F\left(\varepsilon, g, \tilde{Q}_{I}\right) \text {. }
$$

Note that $F$ is a polynomial (and hence, smooth) function of each variable. One can verify that $F\left(0, g^{\lim }, \mathbf{0}\right)=\mathbf{0}$, where

$$
g^{\lim }:=-\left[\begin{array}{c}
X_{L L} \\
X_{I L}
\end{array}\right] \operatorname{diag}^{-1}\left(\mathbb{1}+M_{L} \eta\right) \operatorname{diag}\left(M_{I} \eta\right) Q_{L} .
$$

Moreover, we compute the partial derivative

$$
\frac{\partial F}{\partial g}\left(0, g^{\lim }, \mathbf{0}\right)=\operatorname{diag}(\mathbb{1}+M \tilde{\eta}) \mathcal{L},
$$

which is nonsingular since $\mathcal{L}$ is positive definite and (23) holds. By the implicit function theorem [17], there exist open sets $U_{0} \subset \mathbb{R}$ and $V_{0} \subset \mathbb{R}^{\left|\mathcal{V}_{I}\right|}$, both containing the origin, and a function $G: U_{0} \times V_{0} \rightarrow \mathbb{R}^{\left|\mathcal{V}_{L}\right|+\left|\mathcal{V}_{I}\right|}$,

$$
\left(\varepsilon, \tilde{Q}_{I}\right) \mapsto g=G\left(\varepsilon, \tilde{Q}_{I}\right)=\left[\begin{array}{c}
G_{L}\left(\varepsilon, \tilde{Q}_{I}\right) \\
G_{I}\left(\varepsilon, \tilde{Q}_{I}\right)
\end{array}\right],
$$

such that $F\left(\varepsilon, G\left(\varepsilon, \tilde{Q}_{I}\right), \tilde{Q}_{I}\right)=\mathbf{0}$ for $\varepsilon \in U_{0}$ and $\tilde{Q}_{I} \in V_{0}$. Substituting $g_{I}=G_{I}\left(\varepsilon, \tilde{Q}_{I}\right)$ into the dynamics (25b) we obtain

$$
\begin{aligned}
-\tilde{Q}_{I} & =\varepsilon X_{I I} \dot{\tilde{Q}}_{I}+\varepsilon \dot{G}_{I}\left(\varepsilon, \tilde{Q}_{I}\right) \\
& =\varepsilon\left(X_{I I}+\frac{\partial G_{I}}{\partial \tilde{Q}_{I}}\left(\varepsilon, \tilde{Q}_{I}\right)\right) \dot{\tilde{Q}}_{I} .
\end{aligned}
$$

The matrix $\partial G_{I} / \partial \tilde{Q}_{I}$ is a sub-matrix of $\partial G / \partial \tilde{Q}_{I}$, whose expression is provided by the implicit function theorem as

$$
\frac{\partial G}{\partial \tilde{Q}_{I}}\left(\varepsilon, \tilde{Q}_{I}\right)=-\left(\frac{\partial F}{\partial g}\right)^{-1} \frac{\partial F}{\partial \tilde{Q}_{I}},
$$

which holds in $U_{0} \times V_{0}$ and can be computed by means of simple but lengthy linear algebra operations which we omit, resulting in the expression

$$
\begin{aligned}
\frac{\partial G}{\partial \tilde{Q}_{I}}\left(\varepsilon, \tilde{Q}_{I}\right)= & -\left\{\operatorname{diag}(\mathbb{1}+M \tilde{\eta}) \mathcal{L}+\varepsilon^{2} r\right\}^{-1} \\
& \cdot\left(\left[\begin{array}{c}
\mathbf{0} \\
\operatorname{diag}\left(M_{I} \eta\right)
\end{array}\right]+\varepsilon^{2} s\right),
\end{aligned}
$$

where $r$ and $s$ are polynomial functions in $\varepsilon, \tilde{Q}_{I}$, and $G\left(\varepsilon, \tilde{Q}_{I}\right)$.

Part 3 (Fixed point and its stability): In $U_{0} \times V_{0}$ we have that $\varepsilon, \tilde{Q}_{I}$ and $G\left(\varepsilon, \tilde{Q}_{I}\right)$ are bounded and so are $r$ and $s$. 
Therefore, from (28) we have

$$
\lim _{\varepsilon \rightarrow 0} \frac{\partial G_{I}}{\partial \tilde{Q}_{I}}=-X_{I I} \operatorname{diag}^{-1}\left(\mathbb{1}+X_{I I} C \tilde{\eta}\right) \operatorname{diag}\left(X_{I I} C \tilde{\eta}\right) .
$$

It follows that

$$
\lim _{\varepsilon \rightarrow 0}\left(X_{I I}+\frac{\partial G_{I}}{\partial \tilde{Q}_{I}}\left(\varepsilon, \tilde{Q}_{I}\right)\right)=X_{I I} \operatorname{diag}^{-1}\left(\mathbb{1}+X_{I I} C \eta\right) .
$$

The right-hand side of (29) is invertible. Since the invertibility of the matrix depends continuously on the matrix entries, there exists $\hat{\varepsilon}>0$ such that for all $\varepsilon<\hat{\varepsilon}$ the matrix

$$
X_{I I}+\frac{\partial G_{I}}{\partial \tilde{Q}_{I}}\left(\varepsilon, \tilde{Q}_{I}\right) \text {. }
$$

is also invertible. Thus, for $\varepsilon<\hat{\varepsilon}$, we can now obtain from (26) the explicit dynamical system

$$
\begin{aligned}
\dot{\tilde{Q}}_{I} & =-\frac{1}{\varepsilon}\left(X_{I I}+\frac{\partial G_{I}}{\partial \tilde{Q}_{I}}\left(\varepsilon, \tilde{Q}_{I}\right)\right)^{-1} \tilde{Q}_{I} \\
& :=A\left(\tilde{Q}_{I}\right) \tilde{Q}_{I}:=f\left(\tilde{Q}_{I}\right)
\end{aligned}
$$

where we defined $A$ and $f$ to keep the notation compact. Observe from (30) (or, from (18)) that $\tilde{Q}_{I}=\mathbf{0}$ is the unique fixed point of the dynamics. The Jacobian of the system (30) around $\tilde{Q}_{I}=\mathbf{0}$ is given by

$$
\begin{aligned}
\left.\frac{\partial f\left(\tilde{Q}_{I}\right)}{\partial \tilde{Q}_{I}}\right|_{\tilde{Q}_{I}=0} & =A\left(\tilde{Q}_{I}=\mathbf{0}\right) \\
& =-\frac{1}{\varepsilon}\left(X_{I I}+\frac{\partial G_{I}}{\partial \tilde{Q}_{I}}\left(\varepsilon, \tilde{Q}_{I}=\mathbf{0}\right)\right)^{-1} .
\end{aligned}
$$

Since the inverse of a matrix is continuous with respect to the matrix entries, (29) leads to

$$
\left[\lim _{\varepsilon \rightarrow 0}\left(X_{I I}+\frac{\partial G_{I}}{\partial \tilde{Q}_{I}}(\varepsilon, \mathbf{0})\right)\right]^{-1}=\operatorname{diag}\left(\mathbb{1}+X_{I I} C \eta\right) X_{I I}^{-1} .
$$

The product between a diagonal positive definite matrix and a positive definite matrix has all the eigenvalues with positive real part (see $[18]$ or $[19, \S 6.2]$ ).

As the eigenvalues of a matrix depend continuously on the matrix entries, it is possible to find $\left.\varepsilon^{\max } \in\right] 0, \hat{\varepsilon}[$ such that, for all $\varepsilon<\varepsilon^{\max }$, the Jacobian (32) has all its eigenvalues with negative real part. We can conclude that for such values of $\varepsilon$, the point $\tilde{Q}_{I}=\mathbf{0}$ is a locally exponentially stable fixed point for (26). It follows that the fixed point of (25) is $\tilde{Q}_{I}=\mathbf{0}$, along with

$$
g^{\mathrm{eq}}=G\left(\varepsilon, \tilde{Q}_{I}=\mathbf{0}\right) .
$$

Since $g=G\left(\varepsilon, \tilde{Q}_{I}\right)$ is a continuous function of $\tilde{Q}_{I}$, the local exponential stability of $\tilde{Q}_{I}=\mathbf{0}$ implies the local exponential stability of $g^{\mathrm{eq}}$. This in turn is equivalent to the local exponential stability of the fixed point (21) expressed in terms of the original varibles $E_{L}$ and $E_{I}$. Finally, the limit (22) follows from

$$
\lim _{\varepsilon \rightarrow 0} g^{\mathrm{eq}}=\lim _{\varepsilon \rightarrow 0} G\left(\varepsilon, \tilde{Q}_{I}=\mathbf{0}\right)=G(0, \mathbf{0})=g^{\lim } .
$$

This completes the proof of Theorem III.2.

\section{NUMERICAL STUDY}

In this section we test the results obtained in Theorem III.2 on an islanded version of the standard IEEE 37 distribution network. The line susceptances for this network vary in the range $[-0.5 \mathrm{H},-10 \mathrm{H}]$ with $R / X$ ratios of approximately one, while the reactive power demands vary for each load in the interval [ $-30 \mathrm{kvar},-70 \mathrm{kvar}]$. The sources in this

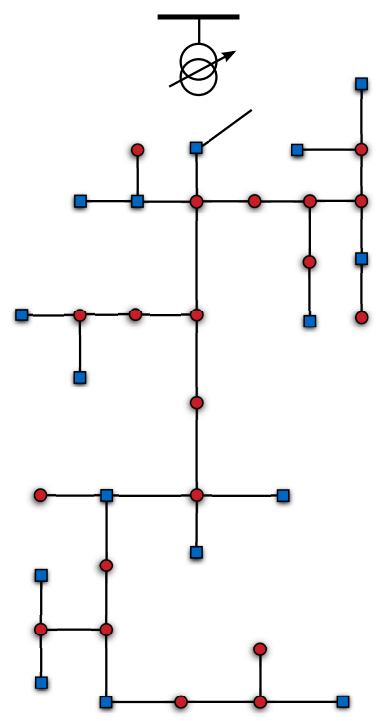

Fig. 2. Islanded IEEE 37 bus distribution network containing loads $\bullet$ and inverters -

network are DC/AC inverters, whose voltage magnitudes are governed by the quadratic droop controller (14). We simulate the resulting differential-algebraic system (19) for different values of $\tilde{E}_{N}$ and study:

a) the threshold $\tilde{E}_{N}^{\min }$ above which the fixed point (21) exists and is stable, and

b) the accuracy of the approximated fixed point expression (21). We consider two variations on the approximation presented in (21):

B1: $\quad$ we set $g^{\mathrm{eq}}=g^{\lim }$ in $(21) ;$
B2: $\quad$ we set $g^{\mathrm{eq}}=\mathbf{0}$ in $(21)$.

To quantify the error between the true fixed point $E_{\text {nonlin }}^{e q}$ of the nonlinear system and the approximations given by $\mathbf{B} 1$ and $\mathbf{B 2}$, we introduce the relative approximation errors

$$
\delta_{i}:=\frac{\left\|E_{\text {nonlin }}^{e q}-E_{\text {approx }, \mathbf{B} i}^{e q}\right\|_{\infty}}{E_{N}}, \quad i \in\{1,2\} .
$$

We note that, in studying the accuracy of Theorem III.2, we will implicitly also study the accuracy of the preliminary result Theorem II.2, as the former depends on the latter. The threshold $\tilde{E}_{N}^{\min }$ above which a stable high-voltage fixed point exists was found by simulation to be roughly $860 \mathrm{~V}$, well below the muli-kV range in which the system is operated. Figure 3 reports the relative approximation errors $\delta_{1}$ and $\delta_{2}$ for the approximations B1 and B2 in the IEEE 37 network, for different values of $\tilde{E}_{N}$. 


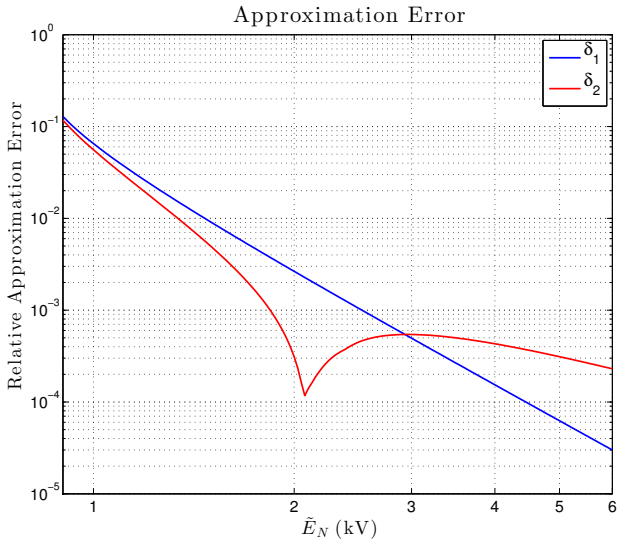

Fig. 3. Relative approximation error for simulation of the IEEE 37 bus distribution network as a function of the nominal network voltage. The scales on both axes are logarithmic.

Note first that both relative approximation errors decreases rapidly as $\tilde{E}_{N}$ grows. In particular at the $4.8 \mathrm{kV}$ nominal operating voltage of the network, the relative error using both approximations is below $0.1 \%$, with the accuracy of $\mathbf{B 1}$ being below $0.01 \%$. For large values of $\tilde{E}_{N}$ the approximation $\mathbf{B 1}$ is more accurate, so exploiting the knowledge of $g^{\lim }$ leads to a better approximation for practical operational region. The curious and smooth behavior of the relative approximation error - and the relation of this behavior to the bounds in (13) — is a subject of future research.

\section{CONCLUSIONS}

In this work we have presented novel analytic expressions for the approximate solution of the decoupled reactive power flow equations. Aside from the clear application in transmission networks, we have demonstrated the flexibility of our result by using it to study the behavior of droopcontrolled inverters in an islanded microgrid. Through simulation, we have demonstrated that our results are practical and extremely accurate in real power networks. Future work in this direction seeks to examine analytically the results of Figure 3, and to further relax the assumption of small angular differences. We further envision an extensive set of case studies, with the goal of demonstrating conclusively the usefulness of this approximation in power system planning and operation.

\section{REFERENCES}

[1] A. Araposthatis, S. Sastry, and P. Varaiya, "Analysis of powerflow equation," International Journal of Electrical Power \& Energy Systems, vol. 3, no. 3, pp. 115-126, 1981.

[2] I. Dobson, "Observations on the geometry of saddle node bifurcation and voltage collapse in electrical power systems," IEEE Transactions on Circuits and Systems I: Fundamental Theory and Applications, vol. 39, no. 3, pp. 240-243, 1992.

[3] H. Chiang and M. Baran, "On the existence and uniqueness of load flow solution for radial distribution power networks," IEEE Transactions on Circuits and Systems, vol. 37, no. 3, pp. 410-416, 1990.

[4] F. Wu and S. Kumagai, "Steady-state security regions of power systems," IEEE Transactions on Circuits and Systems, vol. 29, no. 11, pp. 703-711, 1982.
[5] J. Thorp, D. Schulz, and M. Ilić-Spong, "Reactive power-voltage problem: conditions for the existence of solution and localized disturbance propagation," International Journal of Electrical Power \& Energy Systems, vol. 8, no. 2, pp. 66-74, 1986.

[6] M. Ilić, "Network theoretic conditions for existence and uniqueness of steady state solutions to electric power circuits," in IEEE International Symposium on Circuits and Systems, San Diego, CA, USA, May 1992, pp. 2821-2828.

[7] S. Bolognani and S. Zampieri, "A distributed control strategy for reactive power compensation in smart microgrids," IEEE Transactions on Automatic Control, vol. 58, no. 11, 2013, to Appear.

[8] B. C. Lesieutre, P. W. Sauer, and M. A. Pai, "Existence of solutions for the network/load equations in power systems," IEEE Transactions on Circuits and Systems I: Fundamental Theory and Applications, vol. 46, no. 8, pp. 1003-1011, 1999.

[9] P. Kundur, Power System Stability and Control. McGraw-Hill, 1994.

[10] F. Dörfler and F. Bullo, "Novel insights into lossless AC and DC power flow," in IEEE Power \& Energy Society General Meeting, Vancouver, BC, Canada, July 2013, to appear.

[11] J. Machowski, J. W. Bialek, and J. R. Bumby, Power System Dynamics, 2nd ed. Wiley, 2008.

[12] J. A. P. Lopes, C. L. Moreira, and A. G. Madureira, "Defining control strategies for microgrids islanded operation," IEEE Transactions on Power Systems, vol. 21, no. 2, pp. 916-924, 2006.

[13] M. C. Chandorkar, D. M. Divan, and R. Adapa, "Control of parallel connected inverters in standalone AC supply systems," IEEE Transactions on Industry Applications, vol. 29, no. 1, pp. 136-143, 1993.

[14] J. W. Simpson-Porco, F. Dörfler, and F. Bullo, "Voltage stabilization in microgrids using quadratic droop control," in IEEE Conf. on Decision and Control, Florence, Italy, Dec. 2013, to appear.

[15] R. A. Horn and C. R. Johnson, Topics in Matrix Analysis. Cambridge University Press, 1994.

[16] C. Meyer and M. Stadelmaier, "Singular M-matrices and inverse positivity," Linear Algebra and its Applications, vol. 22, pp. 139-156, 1978

[17] R. Abraham, J. E. Marsden, and T. S. Ratiu, Manifolds, Tensor Analysis, and Applications, 2nd ed., ser. Applied Mathematical Sciences. Springer, 1988, vol. 75.

[18] D. Carlson and H. Schneider, "Inertia theorems for matrices: The semidefinite case," Journal of Mathematical Analysis and Applications, vol. 6, pp. 430-446, 1963.

[19] H. Goldstein, C. Poole, and J. Safko, Classical Mechanics, 3rd ed. Addison-Wesley, 2000. 